\title{
Trends and determinants of vaccination among children aged 06-59 months in Bangladesh: country representative survey from 1993 to 2014
}

Md. Moyazzem Hossain ${ }^{1,2^{*}}$ (D, Md. Abdus Sobhan ${ }^{3}$, Azizur Rahman $^{4^{*}}$, Sanzida Sharmin Flora ${ }^{5}$ and Zahida Sultana Irin ${ }^{6}$

\begin{abstract}
Background: Vaccination has important consequences for childhood development, mortality, and inequalities in health and well-being. This research explores the trend of vaccinations coverage from 1993 to 2014 and determines the significant factors for vaccinations coverage in Bangladesh, considering geospatial, socioeconomic, and demographic characteristics.

Methods: This study uses a secondary dataset extracted from the Bangladesh Demographic and Health Survey (BDHS) from 1992 to 93 to 2014. The association between selected independent variables and vaccination coverage of children was examined through the Chi-square test. In addition, unadjusted and adjusted logistic regression approaches were applied to determine the effects of covariates on vaccination status by using the BDHS-2014 dataset.

Results: The results reveal that the trend of the vaccination coverage rate has gradually been increased over the study period. The coverage rate of BCG is observed maximum while the lowest for Measles vaccination among all types of vaccinations. The findings revealed that the significantly lower coverage of all vaccination had been observed in the Sylhet region. Children of higher educated mothers (OR 10.21; Cl: 4.10-25.37) and father (OR 8.71; $\mathrm{Cl}$ : 4.03-18.80), born at health facilities (OR 4.53; Cl: 2.4-8.55) and whose mother has media exposure (OR 3.20; Cl: 2.22-4.60) have more chance of receiving BCG vaccine. For DPT vaccination coverage, there is a significant difference from children whose mothers have primary (OR 1.7; Cl: 1.35-2.15), secondary (OR 3.5; Cl: 2.75-4.45), and higher (OR 9.6; Cl: 5.28-17.42) educational qualification compared to children of illiterate mothers. Findings demonstrated that children born in wealthier households have a higher likelihood of being immunized against DPT, Polio, and Measles vaccination than children born in the poorest households.
\end{abstract}

\footnotetext{
* Correspondence: hossainmm@juniv.edu; m.m.hossain2@newcastle.ac.uk; azrahman@csu.edu.au

'Department of Statistics, Jahangirnagar University, Savar, Dhaka 1342, Bangladesh

${ }^{4}$ School of Computing, Mathematics and Engineering, Charles Sturt University, Wagga Wagga, NSW 2678, Australia

Full list of author information is available at the end of the article
}

(c) The Author(s). 2021 Open Access This article is licensed under a Creative Commons Attribution 4.0 International License, which permits use, sharing, adaptation, distribution and reproduction in any medium or format, as long as you give appropriate credit to the original author(s) and the source, provide a link to the Creative Commons licence, and indicate if changes were made. The images or other third party material in this article are included in the article's Creative Commons licence, unless indicated otherwise in a credit line to the material. If material is not included in the article's Creative Commons licence and your intended use is not permitted by statutory regulation or exceeds the permitted use, you will need to obtain permission directly from the copyright holder. To view a copy of this licence, visit http://creativecommons.org/licenses/by/4.0/ The Creative Commons Public Domain Dedication waiver (http://creativecommons.org/publicdomain/zero/1.0/) applies to the data made available in this article, unless otherwise stated in a credit line to the data. 
Conclusions: The findings reveal that to enhance and make sustainable the overall country's vaccination coverage, we should pay more attention to the mother's education, socioeconomic condition, children's age, birth order number, having media exposure, place of residence, and religion. The authors think that this finding would be helpful to accelerate the achievement target of Sustainable Development Goals (SDGs) for children's health in Bangladesh.

Keywords: Trends in coverage rate, Vaccination, Adjusted logistic regression, BDHS, Bangladesh

\section{Background}

The Expanded Program on Immunization (EPI) was officially established in 1974, with the support of the World Health Organization (WHO), keeping in mind as an important goal of immunizing every child counter to four vaccine-preventable diseases (VPD) (i.e., tuberculosis, poliomyelitis, measles and diphtheria, pertussis, tetanus (DPT)) by 1990 [1]. However, the EPI set the priority for the developing countries since the higher prevalence and inadequate service delivery for immunization are observed in those countries [2]. The immunization programme for children against VPD has been considered one of the best cost-effective programmes to lessen childhood morbidities and mortalities throughout the world [3, 4]. Moreover, vaccination against VPDs averts illness and disability, saving millions of children globally every year [5]. The rates of vaccine-preventable diseases have declined in many regions worldwide in the past few decades, but many children remain unvaccinated [6]. Moreover, every year, nearly 20 million infants do not receive the complete set of recommended vaccines globally [7]. However, over the past decades, a remarkable improvement has been observed in developing the national immunization program [8]. According to a recent study, the supply chain of lifesaving vaccination has lately been disrupted in several nations due to the lockdown measures adopted by most countries due to COVID-19 [9].

Vaccines have saved uncountable lives, minimized the incidence of polio globally by $99 \%$ and reduced illness, disability, and death from several diseases like tetanus, diphtheria, measles, $H$. influenzae type b disease, whooping cough and epidemic meningococcal A meningitis [10]. Therefore, immunization should be considered a fundamental element of the human right to health and must play a vital role by the individual, community, and government of a country. Like other countries, the government of Bangladesh had originated EPI with the backing of UNICEF and WHO via several activities from 1979, with the overall objective to immunize all children by 1990 to prevent the VPDs and eliminate poliomyelitis $[1,11]$. As a result, Bangladesh has experienced notable enhancements in increasing immunization coverage and a noteworthy contribution to the lessening of childhood morbidity, mortality and also continuing its polio-free status [12]. However, despite declining the child mortality rate, the country included in the list of the top ten countries with the highest childhood mortality globally [13].

In several countries, it has been identified that vaccination coverage is associate with socioeconomic and demographic characteristics. Previous studies have been reported that parental education level [4, 14-17], age of child $[16,18,19]$, employment status as well as workplace [14, 16, 17], religion [20], ethnic origin [21], sex of child [22], poverty [23-27], and distance to healthcare facilities $[14,16,20,24,26,27]$ are linked to vaccination coverage. However, significant differences often exist between countries [20]. Moreover, the findings of several previous studies have generated evidence about the socio-demographic factors related to full vaccination among children [28-30]. Furthermore, several existing studies have focused on specified geographical settings, age groups, or ethnic groups rather than the countrywide setting and have not identified the determinants of individual vaccines separately $[2,3,29-34]$. The authors were motivated to fill the gap of finding out the potential determinants of individual vaccination coverage of BCG, DPT, Polio, and Measles in Bangladesh. Therefore, this study demonstrates the trend from 1993 to 2014 and sought out the influential determinant to capture the full vaccination status using the 2014 Bangladesh Demographic and Health Survey (BDHS-2014) dataset.

\section{Methods}

Data sources, study design and participants

To bear in mind the first objectives of this study, i.e., for trend estimation purposes, the authors used seven nationally representative Bangladesh Demographic Health Survey (BDHS) data (1993-1994, 1996-1997, 1999_ 2000, 2004, 2007, 2011 and 2014) and finally, to identify the significant determinants of child vaccination coverage against BCG, DPT, Polio and Measles, this study uses the BDHS-2014 data set. In BDHS-2014, the sampling frame was used as a complete list of enumeration areas (EAs) covering the country prepared by the Bangladesh Bureau of Statistics for the 2011 population census of the People's Republic of Bangladesh. An EA is a geographic area covering, on average, 113 households. However, the 2014 BDHS sample was stratified and 
selected in two stages. In the first stage, 600 EAs were selected with probability proportional to the EA size. A fixed number of 30 households per cluster was selected in the second stage selection with an equal probability from the newly created household listing. The data sets were filtered with the inclusion criteria of infants aged between six and 59 months. In addition, the small amount of system missing observations was discarded from the dataset. In order to find out the trend of vaccinations mentioned above, the number of children was 2387, 4468, 5117, 5220, 4614, 6715 and 6246 for BDHS surveys 1993-1994, 1996-1997, 1999-2000, 2004, 2007, 2011 and 2014 respectively. However, the findings of the subsequent analysis were based on 6246 children. The detailed data collection procedures are available on the report of the respective years [35-41].

\section{Study variables}

This study considers four vaccination, i.e., BCG, DPT, Polio, and Measles coverage in Bangladesh. The outcome variable of interest in this study is whether a child received the selected vaccination or not. In this case, if a child received the vaccine, then it is coded as " 1 " represents "yes", and otherwise, it is coded as " 0 " indicates "no". Several demographic, socioeconomic and spatial variables were included in the analysis as predictors of a child's vaccination status. The predictor variables include place of residence (urban or rural), region (Barisal, Chittagong, Dhaka, Khulna, Rajshahi, Rangpur, and Sylhet), religion (Muslim or Non-Muslim), mother's age, mother's education level (no education, primary, secondary and higher) and working status (no, yes), father's education level (no education, primary, secondary and higher) and occupation (farming, non-farming, professionals, business, unemployed and others), and wealth index (poorest, poorer, middle, richer, and richest). The selection of variables used in this study was motivated by the availability in the BDHS dataset and self-efficacy and guided by relevant literature. The Expanded

Table 1 Schedule of the Expanded Program on Immunizations in Bangladesh

\begin{tabular}{ll}
\hline Vaccine & Recommended age period \\
\hline Bacille Calmette Guerin (BCG) & At birth/0 day \\
Pentavalent 1 & 42 days \\
Pentavalent 2 & 70 days \\
Pentavalent 3 & 98 days \\
Oral polio vaccine (OPV) 1 & 42 days \\
Oral polio vaccine (OPV) 2 & 70 days \\
Oral polio vaccine (OPV) 3 & 98 days \\
Measles & 273 days \\
\hline
\end{tabular}

Program on Immunizations (EPI) schedule and timeliness in Bangladesh are given in Table 1.

\section{Ethics}

This research is based on publicly available datasets from the DHS repository removed from any identifiable information. Also, because the data were completely anonymous, the authors did not need to seek additional ethical approval before using them. However, the authors complete the registration process and permission was granted to download and use the datasets.

\section{Statistical analysis}

The trend is illustrated by the line graph over the period of study. The bivariate associations were investigated through the Chi-square test. The modeling was done using the logistic regression model (LRM) to estimate the association's strength and the contribution of the independent variables to the variation of the dependent variable by estimating the odds ratios (95\% confidence interval) and the coefficient of the model, respectively. We run four LRM for BCG, DPT, Polio, and Measles separately. The data processing and analyses were done using MS-Excel, STATA version 14, and IBM SPSS v25.

The logistic regression model can be expressed as,

$$
\operatorname{Pr}\left(\mathrm{Y}_{i}=1\right)=\frac{\exp \left(\mathrm{X}_{i} \beta\right)}{1+\exp \left(\mathrm{X}_{i} \beta\right)}
$$

where, $Y_{i}$ is a binary variable that takes a value of ' 1 ' if the respondent received the vaccination and ' 0 ' otherwise, $X_{i}$ is a vector of independent variables and $\beta$ is a vector of unknown parameters which contains the intercept parameter and the regression parameters associated with a set of covariates used in the study.

The fitted form of the model can be defined as,

$$
\ln \left[\frac{\hat{P}}{1-\hat{P}_{i}}\right]=\hat{\beta}_{0}+\hat{\beta}_{1} X_{1}+\ldots+\hat{\beta}_{k} X_{k}
$$

where, $\hat{\beta}_{l}(l=0,1,2, \ldots, k)$ represents the estimated regression coefficient of the $l^{\text {th }}$ independent variable in the study [42].

The $p$-value of the Chi-square test for measuring association among different characteristics and vaccination received by children aged 06-59 months are presented in Fig. 2. Variables significant at least once are included in the logistic regression models as covariates.

\section{Results}

The proportion of various implemented vaccination by survey years are presented in Table 2. The results revealed that children's rate of BCG vaccinations had been increased from 86.0 to $97.1 \%$ from 1994 to 2014 . Also, the rate of DPT vaccination coverage increased about 
Table 2 Trend of receiving specific vaccination by children in Bangladesh, BDHS-1992 to BDHS-2014

\begin{tabular}{|c|c|c|c|c|c|c|c|c|c|c|c|c|c|c|}
\hline \multirow{2}{*}{$\begin{array}{l}\text { Received } \\
\text { vaccination }\end{array}$} & \multicolumn{2}{|c|}{ 1993-94 } & \multicolumn{2}{|c|}{ 1996-97 } & \multicolumn{2}{|c|}{ 1999-2000 } & \multicolumn{2}{|c|}{2004} & \multicolumn{2}{|c|}{2007} & \multicolumn{2}{|c|}{2011} & \multicolumn{2}{|c|}{2014} \\
\hline & $\%$ & $\begin{array}{l}\text { No. of } \\
\text { Child }\end{array}$ & $\%$ & $\begin{array}{l}\text { No. of } \\
\text { Child }\end{array}$ & $\%$ & $\begin{array}{l}\text { No. of } \\
\text { Child }\end{array}$ & $\%$ & $\begin{array}{l}\text { No. of } \\
\text { Child }\end{array}$ & $\%$ & $\begin{array}{l}\text { No. of } \\
\text { Child }\end{array}$ & $\%$ & $\begin{array}{l}\text { No. of } \\
\text { Child }\end{array}$ & $\%$ & $\begin{array}{l}\text { No. of } \\
\text { Child }\end{array}$ \\
\hline $\mathrm{BCG}$ & 86.0 & 2058 & 88.1 & 3940 & 90.2 & 4618 & 93.6 & 4887 & 96.1 & 4433 & 97.2 & 6525 & 97.1 & 6064 \\
\hline DPT 3 & 68.0 & 1623 & 74.6 & 3331 & 72.0 & 3684 & 82.9 & 4328 & 89.5 & 4129 & 93.1 & 6249 & 92.4 & 5773 \\
\hline POLIO 3 & 68.9 & 1645 & 64.8 & 2894 & 71.7 & 3659 & 85.0 & 4437 & 90.4 & 4171 & 93.4 & 6270 & 93.0 & 5807 \\
\hline MEASLES & 72.7 & 1733 & 76.8 & 3416 & 73.8 & 3778 & 80.1 & 4180 & 84.7 & 3909 & 89.7 & 6025 & 88.0 & 5484 \\
\hline
\end{tabular}

$35 \%$ from 1994 to 2014 . The rate of Polio vaccination coverage was $68.9 \%$ in 1994 and reached $93.0 \%$ in 2014 , and findings depict that the prevalence of Measles vaccination coverage is increased from 72.7 to $88 \%$ over the study period. About 24 percentage point has been increased for a total dose of DPT and Polio over the study period. The highest vaccination coverage rate has been observed for BCG (97.2\%) and subsequently Polio (93.4\%), DPT (93.1\%), and Measles (89.7\%) in 2011 (Table 2).

The BCG vaccination coverage was relatively high compared to other vaccines. Therefore, it is seen that the prevalence of BCG vaccination is increasing gradually over the study period. However, a similar pattern is observed for DPT and Measles vaccines. After 1996-97, the rate is dropped down slightly. However, after the year 2000, we observed an increasing trend for DPT and Measles vaccinations. In the case of Polio, the lowest percentage of receiving the vaccine is observed in 199697, and since then, a gradual upward trend is observed for the remaining survey years (Fig. 1).

Results presented in Supplementary Table 1 disclosed the per cent distribution of various vaccination in light of different influential socioeconomic and demographic factors. Though there has been an upward trend for implementing various vaccination, however, the discrepancy is observed in urban and rural settings over the study period. Moreover, it has been found that in rural areas, 3.9 percentage points for BCG and polio, 2.3 percentage points for DPT/ Pentavalent, 4.1 percentage points for measles have been more increased than urban areas. Figure 2 presents the frequency of significant variables identified by the Chi-square test between variables from existing literature review and various vaccination coverage on the children aged 06-59 months. A detailed description of results for each vaccination coverage is mention in the subsequent sections.

\section{BCG vaccination}

The findings depict that the urban/rural differentials in BCG vaccination coverage decreased from 5.1 percentage points in 1994 to 1.2 percentage points in 2014 (Supplementary Table 1), and there is a significant difference in the unadjusted odds ratio (OR) 1.45 (CI: 1.05-2.02) (Table 3). All regions except the Sylhet have the same situation for BCG vaccination in 2014. The highest (16.5\%) percentage point of the prevalence of BCG vaccination coverage has been increased in the Chittagong region and lowest (3.5\%) percentage point in the Barisal region (Supplementary Table 1). Although coverage variations among different regions have been decreased, there is still a significant difference in

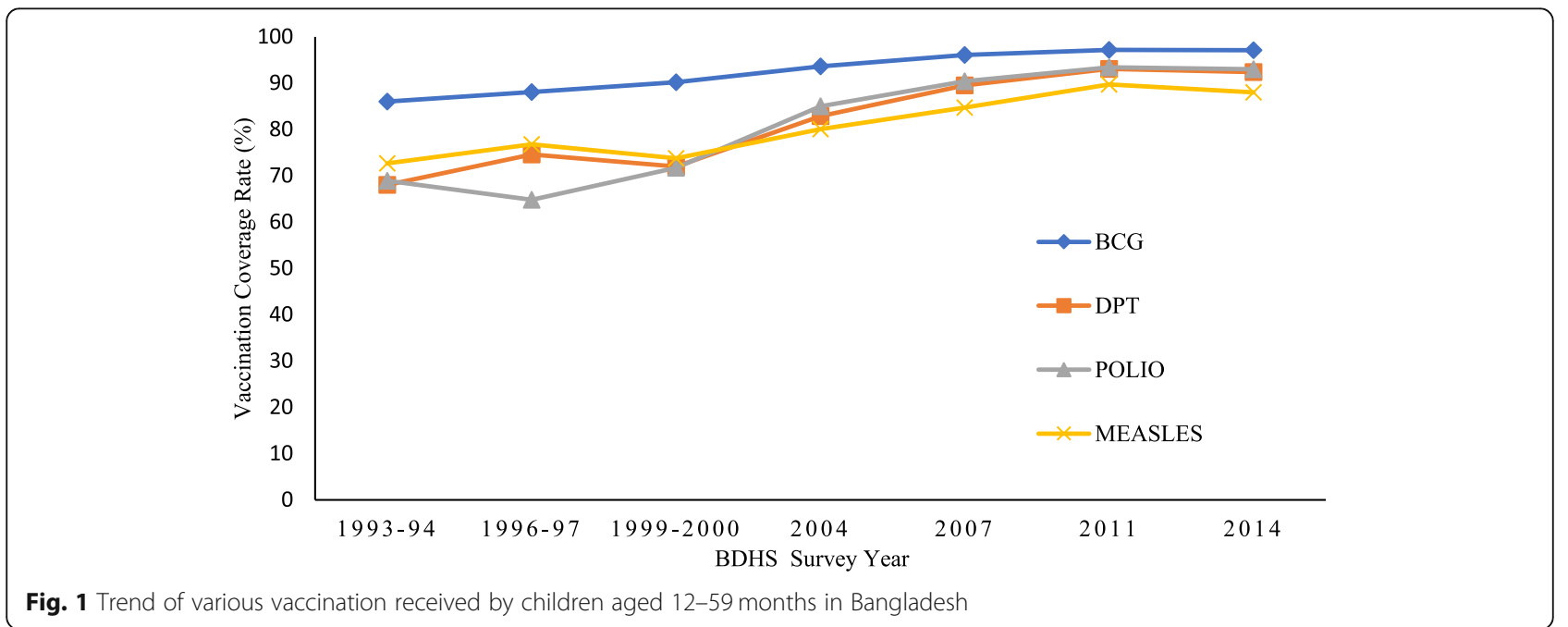




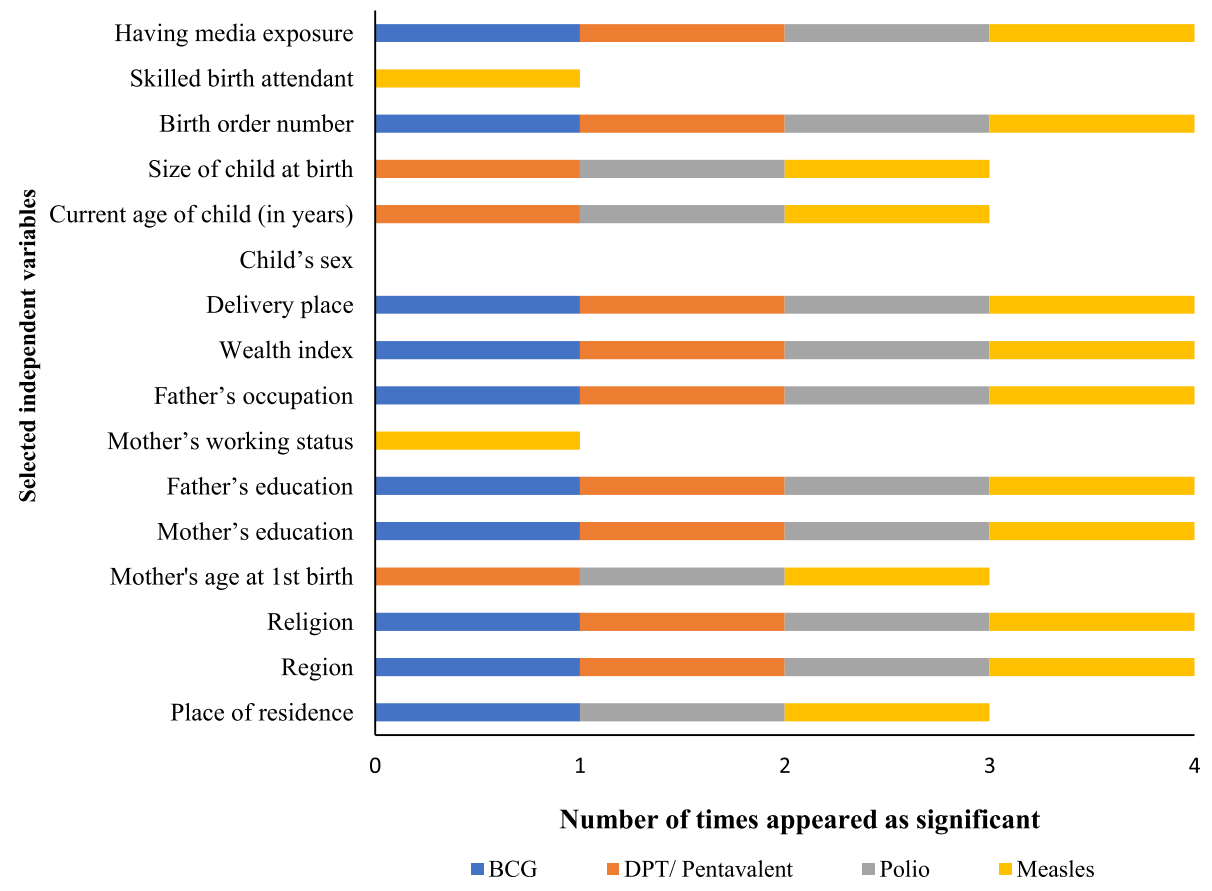

Fig. 2 Number of times appeared significantly $(p<0.05)$ associated independent variables with various vaccinations, BDHS-2014

vaccination rates in the Sylhet region compared to other areas. The difference in BCG vaccination rates between Muslim and non-Muslim children decreased from 9.2\% in 1994 to $1.6 \%$ in 2014 . Also, there is a difference with OR 2.47 (CI: 1.15-5.30) in 2014 among Muslim and non-Muslim children (Table 3). The highest proportion of children have been BCG immunized whose mothers' age at first birth is 25-29 years (Supplementary Table 1).

The proportion of vaccination coverage during the study years has been increased with the increases of mothers' or/and fathers' education levels. An increment is observed in BCG vaccination coverage from illiterate mothers to higher educated mothers (Supplementary Table 1). Children of higher educated mothers have ten times (OR 10.21; CI: 4.10-25.37) more chance to be immunized compared to the children of illiterate mothers. Also, the likelihood of receiving the BCG vaccine is eight times (OR 8.71; CI: 4.03-18.80) more whose father is higher educated compared to illiterate father (Table 3). There exists an almost similar condition of vaccination implementation for employed and non-employed mothers. The child's sex and current age, size of child at birth, and skilled birth attendant do not favor making the difference of BCG vaccination execution rate. Moreover, the BCG vaccination has been less implemented on the children whose fathers are farmers rather than other occupations. Children born in a household with higher socio-economic status had a significantly higher chance of being BCG immunized than children in a poorer household. Delivery at health facilities and having media exposure have shown the more vaccine implementation rate over the study period (Supplementary Table 1). The children have a chance of being 4.5 times (OR 4.53; CI: 2.4-8.55) more BCG vaccinated if they were born at health facilities and of being 3.2 times (OR 3.20; CI: 2.22-4.60) more if their mothers' have media exposure compared to their counterparts.

\section{DPT/ pentavalent vaccination}

This study results disclosed that the proportion of DPT vaccinated children increased 72.7 to $94.6 \%$ from 1994 to 2014 in urban areas while from 67.5 to $91.7 \%$ from 1994 to 2014 in rural areas, but urban/rural differentials are not significant (Supplementary Table 1). The current vaccination coverage status of all regions except the Sylhet division has gradually increased over the study period. However, there is significantly lower coverage of DPT vaccination in Sylhet than in the other regions of Bangladesh. It has been seen that the vaccine coverage on Muslim children is lower than the non-Muslim children from 1994 to 2011, but an equal rate of coverage has been observed in the recent survey. Also, the DPT vaccine implementation rate is higher with the increases of mothers' age at the 1st birth. Although the proportion of DPT immunized children with illiterate mothers increased about 25\% from 1994 to 2014 (Supplementary Table 1). Still, there is a significant difference between children with primary (OR 1.7; CI: 1.35-2.15), secondary (OR 3.5; CI: 2.75-4.45), and higher (OR 9.6; CI: 5.2817.42) educated mothers. Similarly, the vaccination 
Table 3 Results of logistic regression for BCG, DPT, Polio, and Measles, BDHS-2014

\begin{tabular}{|c|c|c|c|c|c|c|c|c|}
\hline \multirow[t]{2}{*}{ Characteristics } & \multicolumn{2}{|l|}{ BCG } & \multicolumn{2}{|c|}{ DPT/ Pentavalent dose 3} & \multicolumn{2}{|l|}{ Polio dose 3} & \multicolumn{2}{|l|}{ Measles } \\
\hline & Crude OR (Cl) & $\begin{array}{l}\text { Adjusted OR } \\
\text { (CI) }\end{array}$ & $\begin{array}{l}\text { Crude OR } \\
\text { (CI) }\end{array}$ & $\begin{array}{l}\text { Adjusted OR } \\
\text { (CI) }\end{array}$ & Crude OR (CI) & $\begin{array}{l}\text { Adjusted OR } \\
\text { (CI) }\end{array}$ & Crude OR (Cl) & $\begin{array}{l}\text { Adjusted OR } \\
\text { (CI) }\end{array}$ \\
\hline \multicolumn{9}{|c|}{ Place of residence } \\
\hline Urban & $1.45(1.05,2.02)^{*}$ & $\begin{array}{l}0.80(0.45 \\
1.40)\end{array}$ & - & - & $\begin{array}{l}1.26(1.01 \\
1.56)^{*}\end{array}$ & $\begin{array}{l}0.64(0.45 \\
0.90)^{*}\end{array}$ & $\begin{array}{l}1.24(1.05 \\
1.46)^{*}\end{array}$ & $0.76(0.58,1.0)$ \\
\hline Rural & Ref & Ref & - & - & Ref & Ref & Ref & Ref \\
\hline \multicolumn{9}{|l|}{ Region } \\
\hline Barisal & Ref & Ref & Ref & Ref & Ref & Ref & Ref & Ref \\
\hline Chittagong & $0.82(0.47,1.43)$ & $\begin{array}{l}0.68(0.27 \\
1.69)\end{array}$ & $\begin{array}{l}0.85(0.59 \\
1.22)\end{array}$ & $\begin{array}{l}0.78(0.46 \\
1.32)\end{array}$ & $\begin{array}{l}0.93(0.63 \\
1.36)\end{array}$ & $\begin{array}{l}0.89(0.52 \\
1.5)\end{array}$ & $\begin{array}{l}1.08(0.81 \\
1.44)\end{array}$ & $\begin{array}{l}0.93(0.61 \\
1.42)\end{array}$ \\
\hline Dhaka & $1.57(0.82,2.97)$ & $\begin{array}{l}1.07(0.38 \\
2.98)\end{array}$ & $\begin{array}{l}1.37(0.91 \\
2.05)\end{array}$ & $\begin{array}{l}1.10(0.62, \\
1.94)\end{array}$ & $\begin{array}{l}1.41(0.93 \\
2.15)\end{array}$ & $\begin{array}{l}1.15(0.65, \\
2.03)\end{array}$ & $\begin{array}{l}1.34(0.99 \\
1.83)\end{array}$ & $\begin{array}{l}1.14(0.73 \\
1.78)\end{array}$ \\
\hline Khulna & $1.20(0.61,2.39)$ & $\begin{array}{l}1.27(0.36 \\
4.50)\end{array}$ & $\begin{array}{l}0.99(0.64 \\
1.51)\end{array}$ & $\begin{array}{l}0.73(0.40, \\
1.32)\end{array}$ & $\begin{array}{l}1.01(0.65 \\
1.57)\end{array}$ & $\begin{array}{l}0.87(0.47 \\
1.59)\end{array}$ & $\begin{array}{l}1.23(0.87 \\
1.73)\end{array}$ & $\begin{array}{l}0.86(0.54 \\
1.38)\end{array}$ \\
\hline Rajshahi & $1.58(0.77,3.23)$ & $\begin{array}{l}1.41(0.44 \\
4.60)\end{array}$ & $\begin{array}{l}1.08(0.71 \\
1.64)\end{array}$ & $\begin{array}{l}1.84(0.78, \\
2.83)\end{array}$ & $0.98(0.64,1.5)$ & $\begin{array}{l}1.45(0.77 \\
2.72)\end{array}$ & $\begin{array}{l}1.14(0.83 \\
1.58)\end{array}$ & $\begin{array}{l}1.23(0.76, \\
1.99)\end{array}$ \\
\hline Rangpur & $\begin{array}{l}3.50(1.38 \\
8.81)^{* *}\end{array}$ & 1.0 (empty) & $\begin{array}{l}1.71(1.06 \\
2.74)^{*}\end{array}$ & $\begin{array}{l}4.44(1.87 \\
10.57)^{* *}\end{array}$ & $\begin{array}{l}1.97(1.19 \\
3.25)^{* *}\end{array}$ & $\begin{array}{l}4.55(1.92 \\
10.8)^{* *}\end{array}$ & $\begin{array}{l}1.81(1.26 \\
2.59)^{* *}\end{array}$ & $\begin{array}{l}2.04(1.21 \\
3.46)^{* *}\end{array}$ \\
\hline Sylhet & $\begin{array}{l}0.30(0.18 \\
0.50)^{* * *}\end{array}$ & $\begin{array}{l}0.22(0.09 \\
0.50)^{* * *}\end{array}$ & $\begin{array}{l}0.34(0.24 \\
0.48)^{* * *}\end{array}$ & $\begin{array}{l}0.34(0.21 \\
0.56)^{* * *}\end{array}$ & $\begin{array}{l}0.35(0.25 \\
0.49)^{* * *}\end{array}$ & $\begin{array}{l}0.37(0.22 \\
0.6)^{* * *}\end{array}$ & $\begin{array}{l}0.43(0.33 \\
0.56)^{* * *}\end{array}$ & $\begin{array}{l}0.34(0.22, \\
0.51)\end{array}$ \\
\hline \multicolumn{9}{|l|}{ Religion } \\
\hline Muslim & Ref & Ref & Ref & Ref & Ref & Ref & Ref & Ref \\
\hline Others & $2.47(1.15,5.30)^{*}$ & $\begin{array}{l}1.68(0.57 \\
4.94)\end{array}$ & $\begin{array}{l}1.62(1.08 \\
2.43)^{*}\end{array}$ & $\begin{array}{l}1.36(0.76, \\
2.45)\end{array}$ & $\begin{array}{l}1.63(1.07 \\
2.48)^{*}\end{array}$ & $\begin{array}{l}1.44(0.79 \\
2.64)\end{array}$ & $\begin{array}{l}1.83(1.30 \\
2.57)^{* * * *}\end{array}$ & $\begin{array}{l}2.03(1.23 \\
3.36)^{* *}\end{array}$ \\
\hline \multicolumn{9}{|c|}{ Mother's age 1st birth (years) } \\
\hline$<18$ & - & - & Ref & Ref & Ref & Ref & Ref & Ref \\
\hline $18-24$ & - & - & $\begin{array}{l}1.33(1.1 \\
1.61)^{* *}\end{array}$ & $\begin{array}{l}1.32(0.99, \\
1.76)\end{array}$ & $\begin{array}{l}1.34(1.1 \\
1.63)^{* *}\end{array}$ & $\begin{array}{l}1.28(0.95 \\
1.72)\end{array}$ & $\begin{array}{l}1.46(1.25 \\
1.7)^{* * *}\end{array}$ & $\begin{array}{l}1.14(0.91, \\
1.44)\end{array}$ \\
\hline $25-29$ & - & - & $\begin{array}{l}1.48(0.86 \\
2.54)\end{array}$ & $\begin{array}{l}1.60(0.61, \\
4.22)\end{array}$ & $\begin{array}{l}1.46(0.84 \\
2.55)\end{array}$ & $\begin{array}{l}1.59(0.6 \\
4.19)\end{array}$ & $\begin{array}{l}1.63(1.05 \\
2.54)^{*}\end{array}$ & $\begin{array}{l}1.23(0.62, \\
2.49)\end{array}$ \\
\hline 30 and above & - & - & $\begin{array}{l}5.43(0.74 \\
39.42)\end{array}$ & 1 (empty) & $\begin{array}{l}1.61(0.50 \\
5.20)\end{array}$ & 1 (empty) & $\begin{array}{l}1.76(0.70 \\
4.44)\end{array}$ & 1 (empty) \\
\hline \multicolumn{9}{|c|}{ Mother's education } \\
\hline No education & Ref & Ref & Ref & Ref & Ref & Ref & Ref & Ref \\
\hline Primary & $\begin{array}{l}1.95(1.40 \\
2.73)^{* * *}\end{array}$ & $\begin{array}{l}1.42(0.81 \\
2.49)\end{array}$ & $\begin{array}{l}1.7(1.35 \\
2.15)^{* * *}\end{array}$ & $\begin{array}{l}1.88(1.3, \\
2.72)^{* *}\end{array}$ & $\begin{array}{l}1.58(1.24 \\
2.0)^{* * *}\end{array}$ & $\begin{array}{l}1.73(1.18 \\
2.51)^{* *}\end{array}$ & $\begin{array}{l}1.47(1.21 \\
1.79)^{* * *}\end{array}$ & $\begin{array}{l}1.59(1.16, \\
2.16)^{* *}\end{array}$ \\
\hline Secondary & $\begin{array}{l}4.77(3.29 \\
6.90)^{* * *}\end{array}$ & $\begin{array}{l}2.0(0.96 \\
4.17)\end{array}$ & $\begin{array}{l}3.5(2.75 \\
4.45)^{* * *}\end{array}$ & $\begin{array}{l}2.42(1.55 \\
3.76)^{* * *}\end{array}$ & $\begin{array}{l}3.55(2.76 \\
4.56)^{* * *}\end{array}$ & $\begin{array}{l}2.26(1.43 \\
3.56)^{* * * *}\end{array}$ & $\begin{array}{l}3.34(2.73 \\
4.08)^{* * * *}\end{array}$ & $\begin{array}{l}2.17(1.52 \\
3.11)^{* * *}\end{array}$ \\
\hline Higher & $\begin{array}{l}10.21(4.10,25 \\
37)^{* * *}\end{array}$ & $\begin{array}{l}4.31(0.46 \\
40.3)\end{array}$ & $\begin{array}{l}9.6(5.28, \\
17.42)^{* * *}\end{array}$ & $\begin{array}{l}6.51(2.03 \\
20.89)^{* *}\end{array}$ & $\begin{array}{l}7.33(4.19 \\
12.83)^{* * *}\end{array}$ & $\begin{array}{l}3.27(1.23, \\
8.72)^{*}\end{array}$ & $\begin{array}{l}7.46(4.83 \\
11.51)^{* * *}\end{array}$ & $\begin{array}{l}4.62(2.09, \\
10.25)^{* * *}\end{array}$ \\
\hline \multicolumn{9}{|c|}{ Husband's education } \\
\hline No education & Ref & Ref & Ref & Ref & Ref & Ref & Ref & Ref \\
\hline Primary & $\begin{array}{l}2.43(1.72 \\
3.41)^{* * *}\end{array}$ & $\begin{array}{l}2.05(1.13 \\
3.69)\end{array}$ & $\begin{array}{l}1.64(1.32 \\
2.04)^{* * *}\end{array}$ & $\begin{array}{l}1.35(0.95 \\
1.91)\end{array}$ & $\begin{array}{l}1.59(1.27 \\
1.99)^{* * *}\end{array}$ & $\begin{array}{l}1.34(0.94, \\
1.92)\end{array}$ & $\begin{array}{l}1.53(1.27 \\
1.83)^{* * *}\end{array}$ & $\begin{array}{l}1.23(0.92, \\
1.64)\end{array}$ \\
\hline Secondary & $\begin{array}{l}3.50(2.37 \\
5.16)^{* * *}\end{array}$ & $\begin{array}{l}1.27(0.64 \\
2.52)\end{array}$ & $\begin{array}{l}3.09(2.38 \\
4.0)^{* * *}\end{array}$ & $\begin{array}{l}1.29(0.84 \\
1.98)\end{array}$ & $\begin{array}{l}3.33(2.53 \\
4.39)^{* * *}\end{array}$ & $\begin{array}{l}1.39(0.89 \\
2.17)\end{array}$ & $\begin{array}{l}2.77(2.25 \\
3.41)^{* * * *}\end{array}$ & $\begin{array}{l}1.13(0.81 \\
(1.59)\end{array}$ \\
\hline Higher & $\begin{array}{l}8.71(4.03 \\
18.80)^{* * *}\end{array}$ & $\begin{array}{l}2.47(0.53 \\
11.5)\end{array}$ & $\begin{array}{l}5.81(3.75 \\
9.0)^{* * *}\end{array}$ & $\begin{array}{l}1.79(0.77 \\
4.15)\end{array}$ & $\begin{array}{l}5.11(3.32 \\
7.87)^{* * *}\end{array}$ & $\begin{array}{l}1.54(0.68 \\
3.46)\end{array}$ & $\begin{array}{l}5.15(3.68 \\
7.21)^{* * * *}\end{array}$ & $1.49(0.8,2.77)$ \\
\hline \multicolumn{9}{|c|}{ Mother's working status } \\
\hline $\begin{array}{l}\text { Non- } \\
\text { employment }\end{array}$ & - & - & - & - & - & - & $\begin{array}{l}1.21(1.03 \\
1.43)^{*}\end{array}$ & $1.16(0.9,1.51)$ \\
\hline
\end{tabular}


Table 3 Results of logistic regression for BCG, DPT, Polio, and Measles, BDHS-2014 (Continued)

\begin{tabular}{|c|c|c|c|c|c|c|c|c|}
\hline \multirow[t]{2}{*}{ Characteristics } & \multicolumn{2}{|l|}{ BCG } & \multicolumn{2}{|c|}{ DPT/ Pentavalent dose 3} & \multicolumn{2}{|l|}{ Polio dose 3} & \multicolumn{2}{|l|}{ Measles } \\
\hline & Crude OR (Cl) & $\begin{array}{l}\text { Adjusted OR } \\
\text { (CI) }\end{array}$ & $\begin{array}{l}\text { Crude OR } \\
\text { (CI) }\end{array}$ & $\begin{array}{l}\text { Adjusted OR } \\
\text { (CI) }\end{array}$ & Crude OR (CI) & $\begin{array}{l}\text { Adjusted OR } \\
\text { (CI) }\end{array}$ & Crude OR (Cl) & $\begin{array}{l}\text { Adjusted OR } \\
\text { (CI) }\end{array}$ \\
\hline Employment & - & - & - & - & - & - & Ref & Ref \\
\hline \multicolumn{9}{|l|}{ Father's occupation } \\
\hline Farming & Ref & Ref & Ref & Ref & Ref & Ref & Ref & Ref \\
\hline Non-farming & $1.31(0.91,1.84)$ & $\begin{array}{l}0.72(0.4 \\
1.28)\end{array}$ & $\begin{array}{l}1.22(0.98 \\
1.52)\end{array}$ & $\begin{array}{l}0.94(0.67 \\
1.31)\end{array}$ & $\begin{array}{l}1.22(0.97 \\
1.53)\end{array}$ & $\begin{array}{l}0.92(0.64 \\
1.32)\end{array}$ & $\begin{array}{l}1.15(0.96 \\
1.38)\end{array}$ & $\begin{array}{l}0.99(0.75 \\
1.31)\end{array}$ \\
\hline Professionals & $\begin{array}{l}3.27(1.30 \\
8.20)^{* *}\end{array}$ & $\begin{array}{l}0.36(0.06 \\
2.16)\end{array}$ & $\begin{array}{l}3.7(1.98 \\
6.9)^{* * *}\end{array}$ & $\begin{array}{l}0.77(0.26 \\
2.28)\end{array}$ & $\begin{array}{l}3.41(1.83 \\
6.38)^{* * *}\end{array}$ & $\begin{array}{l}0.94(0.32, \\
2.78)\end{array}$ & $\begin{array}{l}3.97(2.39 \\
6.58)^{* * *}\end{array}$ & $\begin{array}{l}0.96(0.42, \\
2.19)\end{array}$ \\
\hline Business & $1.45(0.97,2.19)$ & $\begin{array}{l}0.56(0.28 \\
1.10)\end{array}$ & $\begin{array}{l}1.43(1.09 \\
1.87)^{*}\end{array}$ & $\begin{array}{l}0.94(0.62, \\
1.44)\end{array}$ & $\begin{array}{l}1.43(1.08 \\
1.89)^{*}\end{array}$ & $\begin{array}{l}0.91(0.59, \\
1.41)\end{array}$ & $\begin{array}{l}1.51(1.21 \\
1.89)^{* * *}\end{array}$ & $0.98(0.7,1.38)$ \\
\hline Unemployed & $1.31(0.17,9.76)$ & 1 (empty) & $\begin{array}{l}0.75(0.26 \\
2.16)\end{array}$ & $\begin{array}{l}0.60(0.12, \\
3.01)\end{array}$ & $0.69(0.24,2.0)$ & $0.50(0.1,2.5)$ & $\begin{array}{l}0.97(0.37 \\
2.55)\end{array}$ & $\begin{array}{l}0.63(0.16 \\
2.49)\end{array}$ \\
\hline Others & $0.50(0.25,1.05)$ & $\begin{array}{l}0.39(0.13 \\
1.21)\end{array}$ & $\begin{array}{l}1.03(0.54 \\
1.96)\end{array}$ & $1.0(0.39,2.56)$ & $\begin{array}{l}1.19(0.59 \\
2.39)\end{array}$ & $\begin{array}{l}1.13(0.41, \\
3.12)\end{array}$ & $\begin{array}{l}1.55(0.84 \\
2.88)\end{array}$ & $\begin{array}{l}1.53(0.65 \\
3.64)\end{array}$ \\
\hline \multicolumn{9}{|l|}{ Wealth index } \\
\hline Poorest & Ref & Ref & Ref & Ref & Ref & Ref & Ref & Ref \\
\hline Poorer & $1.49(1.01,2.17)^{*}$ & $\begin{array}{l}0.84(0.47 \\
1.52)\end{array}$ & $\begin{array}{l}1.69(1.31 \\
2.18)^{* * *}\end{array}$ & $\begin{array}{l}1.56(1.06 \\
2.32)^{*}\end{array}$ & $\begin{array}{l}1.87(1.43 \\
2.45)^{* * *}\end{array}$ & $\begin{array}{l}1.79(1.19 \\
2.69)^{* *}\end{array}$ & $\begin{array}{l}1.62(1.31 \\
2.0)^{* * *}\end{array}$ & $\begin{array}{l}1.4(1.02 \\
1.92)^{*}\end{array}$ \\
\hline Middle & $\begin{array}{l}1.86(1.25 \\
2.77)^{* *}\end{array}$ & $\begin{array}{l}0.88(0.44 \\
1.76)\end{array}$ & $\begin{array}{l}2.26(1.71 \\
2.97)^{* * *}\end{array}$ & $\begin{array}{l}1.45(0.94 \\
2.25)\end{array}$ & $\begin{array}{l}2.51(1.88 \\
3.34)^{* * *}\end{array}$ & $\begin{array}{l}1.7(1.08 \\
2.67)^{*}\end{array}$ & $\begin{array}{l}2.12(1.69 \\
2.66)^{* * *}\end{array}$ & $\begin{array}{l}1.45(1.01 \\
2.06)^{*}\end{array}$ \\
\hline Richer & $\begin{array}{l}2.36(1.54 \\
3.60)^{* * *}\end{array}$ & $\begin{array}{l}0.88(0.39 \\
1.98)\end{array}$ & $\begin{array}{l}2.33(1.77 \\
3.05)^{* * *}\end{array}$ & $\begin{array}{l}1.25(0.76 \\
2.06)\end{array}$ & $\begin{array}{l}2.54(1.91 \\
3.37)^{* * *}\end{array}$ & $\begin{array}{l}1.55(0.93 \\
2.61)\end{array}$ & $\begin{array}{l}2.19(1.75 \\
2.73)^{* * *}\end{array}$ & $\begin{array}{l}1.61(1.07 \\
2.44)^{*}\end{array}$ \\
\hline Richest & $\begin{array}{l}7.89(3.94 \\
15.80)^{* * *}\end{array}$ & $\begin{array}{l}1.82(0.50 \\
6.66)\end{array}$ & $\begin{array}{l}5.18(3.6 \\
7.47)^{* * *}\end{array}$ & $\begin{array}{l}2.13(1.07 \\
4.26)^{*}\end{array}$ & $\begin{array}{l}5.2(3.6 \\
7.53)^{* * *}\end{array}$ & $\begin{array}{l}2.73(1.33 \\
5.6)^{* *}\end{array}$ & $\begin{array}{l}3.98(3.03 \\
5.22)^{* * *}\end{array}$ & $2.60(1.5,4.5)^{* *}$ \\
\hline \multicolumn{9}{|l|}{ Delivery place } \\
\hline At home & Ref & Ref & Ref & Ref & Ref & Ref & Ref & Ref \\
\hline At health facility & $\begin{array}{l}4.53(2.4 \\
8.55)^{* * *}\end{array}$ & $\begin{array}{l}1.74(0.86 \\
3.52)\end{array}$ & $\begin{array}{l}2.25(1.66 \\
3.06)^{* * *}\end{array}$ & $\begin{array}{l}1.08(0.76, \\
1.55)\end{array}$ & $\begin{array}{l}2.22(1.63 \\
3.04)^{* * *}\end{array}$ & $\begin{array}{l}1.13(0.78, \\
1.63)\end{array}$ & $\begin{array}{l}1.9(1.51 \\
2.4)^{* * *}\end{array}$ & $\begin{array}{l}0.94(0.71 \\
1.25)\end{array}$ \\
\hline \multicolumn{9}{|c|}{ Current age of child (in years) } \\
\hline$\leq 1$ & - & - & Ref & Ref & Ref & Ref & Ref & Ref \\
\hline 2 & - & - & $\begin{array}{l}1.42(1.1 \\
1.85)^{* *}\end{array}$ & $\begin{array}{l}1.66(1.26 \\
2.2)^{* * *}\end{array}$ & $\begin{array}{l}1.43(1.1 \\
1.87)^{* *}\end{array}$ & $\begin{array}{l}1.68(1.26 \\
2.25)^{* * *}\end{array}$ & $\begin{array}{l}1.38(1.12 \\
1.7)^{* *}\end{array}$ & $\begin{array}{l}1.56(1.25 \\
1.95)^{* * *}\end{array}$ \\
\hline 3 & - & - & $\begin{array}{l}1.13(0.88 \\
1.44)\end{array}$ & - & $\begin{array}{l}1.24(0.96 \\
1.61)\end{array}$ & - & $\begin{array}{l}1.35(1.09 \\
1.66)^{* *}\end{array}$ & - \\
\hline 4 & - & - & $\begin{array}{l}1.40(1.07 \\
1.82)^{*}\end{array}$ & - & $\begin{array}{l}1.42(1.09 \\
1.86)^{*}\end{array}$ & - & $\begin{array}{l}1.4(1.14 \\
1.73)^{* *}\end{array}$ & - \\
\hline \multicolumn{9}{|l|}{$\begin{array}{l}\text { Size of child at } \\
\text { birth }\end{array}$} \\
\hline Very large & - & - & $\begin{array}{l}1.55(0.6 \\
3.98)\end{array}$ & $\begin{array}{l}0.93(0.34 \\
2.51)\end{array}$ & $\begin{array}{l}1.47(0.57 \\
3.79)\end{array}$ & $\begin{array}{l}0.83(0.30 \\
2.25)\end{array}$ & $\begin{array}{l}1.68(0.74, \\
3.84)\end{array}$ & $\begin{array}{l}1.08(0.46 \\
2.55)\end{array}$ \\
\hline $\begin{array}{l}\text { Larger } \\
\text { thanaverage }\end{array}$ & - & - & $\begin{array}{l}1.12(0.63 \\
1.99)\end{array}$ & $\begin{array}{l}0.79(0.43 \\
1.47)\end{array}$ & $\begin{array}{l}1.03(0.58, \\
1.83)\end{array}$ & $\begin{array}{l}0.72(0.38 \\
1.35)\end{array}$ & $\begin{array}{l}1.09(0.67 \\
1.78)\end{array}$ & $\begin{array}{l}0.86(0.51 \\
1.46)\end{array}$ \\
\hline Average & - & - & $\begin{array}{l}1.68(1.05 \\
2.7)^{*}\end{array}$ & $\begin{array}{l}1.23(0.73 \\
2.05)\end{array}$ & $\begin{array}{l}1.65(1.02, \\
2.68)^{*}\end{array}$ & $\begin{array}{l}1.19(0.71 \\
2.02)\end{array}$ & $\begin{array}{l}1.31(0.88 \\
1.97)\end{array}$ & $1.0(0.65,1.55)$ \\
\hline $\begin{array}{l}\text { Smaller than } \\
\text { average }\end{array}$ & - & - & $\begin{array}{l}0.99(0.57 \\
1.70)\end{array}$ & $\begin{array}{l}0.97(0.54 \\
1.73)\end{array}$ & $1.1(0.63,1.94)$ & $\begin{array}{l}1.08(0.59 \\
1.98)\end{array}$ & $\begin{array}{l}0.85(0.54 \\
1.35)\end{array}$ & $0.82(0.5,1.34)$ \\
\hline Very small & - & - & Ref & Ref & Ref & Ref & Ref & Ref \\
\hline \multicolumn{9}{|l|}{ Birth order number } \\
\hline 1 & $\begin{array}{l}3.72(2.52, \\
5.51)^{* * *}\end{array}$ & $\begin{array}{l}2.38(1.18 \\
4.82)^{*}\end{array}$ & $\begin{array}{l}2.33(1.82 \\
2.99)^{* * *}\end{array}$ & $\begin{array}{l}0.96(0.64 \\
1.45)\end{array}$ & $\begin{array}{l}2.4(1.86 \\
3.11)^{* * *}\end{array}$ & $\begin{array}{l}1.0(0.66, \\
1.52)\end{array}$ & $\begin{array}{l}2.35(1.91 \\
2.89)^{* * * *}\end{array}$ & $1.1(0.78,1.54)$ \\
\hline
\end{tabular}


Table 3 Results of logistic regression for BCG, DPT, Polio, and Measles, BDHS-2014 (Continued)

\begin{tabular}{|c|c|c|c|c|c|c|c|c|}
\hline \multirow[t]{2}{*}{ Characteristics } & \multicolumn{2}{|l|}{ BCG } & \multicolumn{2}{|c|}{ DPT/ Pentavalent dose 3} & \multicolumn{2}{|l|}{ Polio dose 3} & \multicolumn{2}{|l|}{ Measles } \\
\hline & Crude OR (Cl) & $\begin{array}{l}\text { Adjusted OR } \\
\text { (Cl) }\end{array}$ & $\begin{array}{l}\text { Crude OR } \\
\text { (CI) }\end{array}$ & $\begin{array}{l}\text { Adjusted OR } \\
\text { (CI) }\end{array}$ & Crude OR (CI) & $\begin{array}{l}\text { Adjusted OR } \\
\text { (CI) }\end{array}$ & Crude OR (Cl) & $\begin{array}{l}\text { Adjusted OR } \\
\text { (CI) }\end{array}$ \\
\hline 2 & $\begin{array}{l}2.56(1.75 \\
3.74)^{* * *}\end{array}$ & $\begin{array}{l}1.61 \text { (0.81, } \\
3.22)\end{array}$ & $\begin{array}{l}2.02(1.82 \\
2.99)^{* * * *}\end{array}$ & $\begin{array}{l}1.33(0.86, \\
2.05)\end{array}$ & $\begin{array}{l}2.15(1.64, \\
2.8)^{* * *}\end{array}$ & $\begin{array}{l}1.47(0.94 \\
2.3)\end{array}$ & $\begin{array}{l}1.99(1.61 \\
2.46)^{* * *}\end{array}$ & $1.1(0.78,1.56)$ \\
\hline 3 & $\begin{array}{l}1.64(1.09, \\
2.45)^{* * *}\end{array}$ & $\begin{array}{l}0.67(0.37 \\
1.21)\end{array}$ & $\begin{array}{l}1.67(1.24 \\
2.23)^{* *}\end{array}$ & $\begin{array}{l}0.96(0.62, \\
1.47)\end{array}$ & $\begin{array}{l}1.65(1.22, \\
2.22)^{* * *}\end{array}$ & $\begin{array}{l}0.98(0.63 \\
1.53)\end{array}$ & $\begin{array}{l}1.67(1.31 \\
2.13)^{* * * *}\end{array}$ & $\begin{array}{l}0.96(0.67 \\
1.37)\end{array}$ \\
\hline 4 and above & Ref & Ref & Ref & Ref & Ref & Ref & Ref & Ref \\
\hline \multicolumn{9}{|c|}{ Skilled birth attendant } \\
\hline No & - & - & - & - & - & - & $\begin{array}{l}1.37(1.01 \\
1.85)^{*}\end{array}$ & $\begin{array}{l}1.27(0.91 \\
1.76)\end{array}$ \\
\hline Yes & - & - & - & - & - & - & Ref & Ref \\
\hline \multicolumn{9}{|c|}{ Having media exposure } \\
\hline No & Ref & Ref & Ref & Ref & Ref & Ref & Ref & Ref \\
\hline Yes & $\begin{array}{l}3.20(2.22 \\
4.60)^{* * * *}\end{array}$ & $\begin{array}{l}2.39(1.17 \\
4.90)\end{array}$ & $\begin{array}{l}2.35(1.89 \\
2.91)^{* * *}\end{array}$ & $\begin{array}{l}1.49(1.0 \\
2.23)^{*}\end{array}$ & $\begin{array}{l}2.37(1.9 \\
2.97)^{* * *}\end{array}$ & $\begin{array}{l}1.46(0.97 \\
2.2)\end{array}$ & $\begin{array}{l}2.13(1.8 \\
2.51)^{* * *}\end{array}$ & $1.2(0.88,1.63)$ \\
\hline
\end{tabular}

${ }^{* \prime} p<0.05^{\prime},{ }^{* * \prime} p<0.011^{\prime},{ }^{* * \prime} p<0.001$; Ref Reference category; OR Odds ratio; Cl 95\% confidence interval

coverage rate of children with illiterate fathers is significantly different (OR 5.81; CI: 3.75-9.0) from children with higher educated fathers (Table 3 ).

The proportion of vaccine implementation is more for non-employed mothers than employed mothers, but the difference is not statistically significant. The DPT vaccination has been more implemented by 3.7 times (OR 3.7; CI: 1.98-6.9) on children whose fathers are professionals than children whose fathers are farmers. Children who lived in a household with a better wealth index have a chance of being more DPT vaccinated than that of poorer households. Vaccination coverage rate has been different between children who were born at health facility and born at home $(p<0.001)$; and mother has media exposure have shown the more (OR 2.35; CI: 1.89-2.91) vaccine implementation rate during the study period (Table 3). No significant pattern (increased or decreased in implementation) was exhibited with the child's current age and skilled birth attendant. DPT vaccination coverage is significantly decreased with higher birth order (Supplementary Table 1).

\section{Polio vaccination}

Table 3 revealed that the Polio vaccine coverage differentials in urban/rural context decreased from $6.4 \%$ in 1994 to $2.5 \%$ in 2014 (Supplementary Table 1). There is a statistically significant difference between urban and rural areas in Polio vaccination coverage (OR 1.26; CI: 1.01-1.56). The highest proportion and rate of change of Polio vaccine implementation exist in the Dhaka region rather than the other regions. The lowest execution of the Polio vaccine is observed in the Sylhet region with an odds ratio of 0.35 (CI: 0.25-0.49) over the study period. The proportion of children have been vaccinated from the non-Muslim community is $63 \%$ (OR 1.63; CI: $1.07-2.48$ ) higher than their Muslim counterpart (Table 3); though the disparity is decreased 10.4 percentage point in 1994 to 0.9 percentage point in 2014 (Supplementary Table 1). Moreover, the Polio vaccination rate is increased with the increases of mothers' age at the 1st birth and demonstrated a significant difference between adolescent mothers and mothers aged 18-24 years at first birth with an odds ratio of 1.34 (CI: 1.24-2.0) (Table 3).

Children with educated parents have a chance of being more Polio immunized compared to the parents with no formal education, and this vaccination possibility is more extensive for higher educated mothers than higher educated fathers with an odds ratio of 7.33 (CI: 4.19-12.83) and 5.11 (CI: 3.32-7.87) respectively. The percentage of polio vaccine implementation is more for non-employed mothers than employed mothers and does not reveal any statistical difference. The proportion of children whose father is a professional has been vaccinated 3.4-fold (CI: 1.83-6.38) more than the children whose father is a farmer (Table 3).

The children born in a household with upper wealth quintiles have significantly more chances of being Polio vaccinated than those with lower wealth quintiles. The proportion (OR 2.2; CI: 1.63-3.04) of vaccination and increasing rate, from $70.6 \%$ in 1994 to $96.2 \%$ in 2014 , of the Polio vaccine, is more for health facility delivery than delivery at home. Furthermore, Polio vaccination is significantly decreased with increases in the birth order number. Although the differentials between having and not having media exposure are decreased over the study period (Supplementary Table 1), children whose mother's having media exposure have been more Polio immunized with an odds ratio of 2.37 (CI: 1.9-2.97) compared to children whose mothers having no media exposure (Table 3 ). 


\section{Measles vaccination}

It has been found that there is a significant difference in implementing the Measles vaccine between urban and rural areas with an odds ratio of 1.24 (CI: 1.05-1.46) (Table 3) though the discrepancy is decreased from $7.2 \%$ in 1994 to $3.1 \%$ in 2014 (Supplementary Table 1). The highest proportion of measles vaccination coverage is in the Khulna region, and the lowest is in the Sylhet region. Also, the lowest increase, $64.9 \%$ in 1997 to $74 \%$ in 2014 of Measles vaccination coverage, occurred in the Sylhet region over the study period compared to the other regions. Measles vaccination coverage is $46 \%$ lower in the Muslim community than their counterparts, but the gap decreased from $13.7 \%$ in 1994 to $3.2 \%$ in 2014 . The highest percentage of vaccination implemented is observed among children whose mothers' age at first birth is 25-29 years over the study time (Supplementary Table 1). Moreover, the vaccination rate is significantly different from children whose mothers were adolescent at their first birth (Table 3).

The Measles vaccination rate of children increased from 65.1 to $79.8 \%$ for illiterate mothers and 66.6 to $80.9 \%$ for illiterate fathers from 1994 to 2014 (Supplementary Table 1 ). Also, there is a significant difference among the children with educated parents, and the rate is increased with increasing the parents' education level. Interestingly, the proportion of measles vaccine implementation is higher (OR 1.21; CI: 1.03-1.43) on the children whose mothers are unemployed and mothers with unskilled birth attendants (OR 1.37; CI: 1.01-1.85). Moreover, children have more chances of being more Measles immunized whose fathers are professional (OR 3.97; CI: 2.39-6.58) and businessmen (OR 1.51; CI 1.21-1.89) than those with fathers involved with farming. Improved socio-economic status leads to a significant increase in the Measles vaccination coverage rate. Having media exposure (OR 2.13; CI: 1.82.51; ref.: having no media exposure) and health facility delivery (OR 1.9; CI: 1.51-2.4; ref.: delivery at home) play a significant role to increase the Measles vaccination coverage in 2014 (Table 3). The percentage of children with measles vaccine immunized is decreasing with the increment of the birth order number.

\section{Discussion}

The vaccine-preventable diseases like pneumonia, diarrhoea, and measles collectively responsible for half of the deaths recorded in 2013 [13]. That is, complete immunization coverage may prevent $50 \%$ of illness and death in developing countries. This study exposes the trend and determining factors of the four vaccinations coverage from 1992 to 2014 in Bangladesh. The logistic regression analysis results revealed that several influencing factors, including administrative region, mother's education, socio-economic status, the current age of children, and having media exposure, have been significant for BCG, DPT/Pentavalent 3, Polio, and Measles vaccine implementation respectively.

This study has revealed that the complete dose of DPT has been increased by about 24 percentage point, from 1994 to 2014 and Polio vaccinations received by children aged 06-59 months were increased from 68.9 to $93.0 \%$ over the period 1994 to 2014 . The rate of BCG vaccinations has been increased from $86.0 \%$ in 1994 to $97.1 \%$ in 2014 and it is increased from 72.7 to $88 \%$ from 1994 to 2014 for measles vaccination. The highest vaccination coverage rate has been observed for BCG and the lowest for Measles vaccination over the period 2000 to 2014 (Fig. 1). The reason might be that the recommended age period of children for BCG to Measles vaccination is increasing while mothers or caregivers have been less concerned about immunization with increasing the children's age and its side effects [43].

The adjusted odds ratio demonstrated that the children born in the Sylhet division were less likely to receive vaccines, whereas children born in Rangpur/ Rajshahi division were more likely to receive vaccines. This scenario is observed for all kinds of vaccination coverage received by children. Similar findings are also observed by other researchers [3, 29, 33, 44]. Moreover, Sheikh et al. (2018) highlighted that the Sylhet division primarily covers a remote hilly and riverine area, and the communication system is more fragile than other regions of the country [33]. The mother's education level has been significantly associated with DPT, Polio, and Measles vaccination coverage. Children whose mothers had completed a primary, secondary, and a higher level of education have been 1.88, 2.42, and 6.51 times more likely to receive DPT vaccine; have been $1.73,2.26$, and 3.27 times more likely to receive Polio vaccine; 1.59 , 2.17 , and 4.62 times more likely to receive Measles vaccine respectively as compared with children whose mothers had no formal education. The significance of a mother's education has also been found in many existing papers of home and abroad [2, 3, 28-30, 32, 33, 44-46]. However, such association has not been observed in scenarios of BCG vaccination though a positive relationship has been seen in the unadjusted odds ratio.

This study demonstrated that children born in households with higher wealth quintiles have significantly higher chances of being immunized against DPT, Polio, and Measles than children born in the poorest wealth quintile households. Similar results have been found in other settings [3, 28, 29, 33, 44-46]. Moreover, no significant association is observed for BCG vaccination after adjusting other variables in the model. Children's current age is determined as another highly significant factor in receiving DPT, Polio, and Measles vaccination. It is observed that the children aged 24 months have $1.66,1.68$, and 1.56 times more likely to immunize 
against DPT, Polio, and Measles vaccination, respectively, compared to children aged 12 months. A positive association between vaccination coverage and children's current age has been seen previously [2, 28, 44]. Our research has also revealed that, after adjusting other variables to the model, lower birth order (AOR 2.38; CI: $1.18,4.82)$ is found as a significant factor to have more likely of receiving BCG vaccination compared to higher birth order $(\geq 4)$ and this result is similar with others $[28$, 44]. Children who lived in a household having media exposure have 1.5 times (AOR 1.49; CI: 1.0, 2.23) more likely to be immunized against DPT, and these findings are supported by the others $[3,44,45]$. The place of residence is a significant factor for receiving Polio vaccination, and the likelihood of receiving the measles vaccine is twice (AOR 2.03; CI: 1.23, 3.36) for non-Muslim compared to their Muslim counterpart. Although the mother's working status has been found insignificantly but interestingly, this contradicts to the findings of other settings that children of unemployed mothers failed to receive timely vaccinations for BCG/measles [33].

\section{Strength and limitation}

The strength of the study is that it is using seven nationally representative demographic and health survey data. Therefore, the authors believe that the findings are representative of a whole nation. Additionally, in this study, we consider four vaccination coverage. Despite these strengths, the inclusion criteria are children aged 06-59 months. The future study should focus on expanding the analysis to include other explanatory variables such as geospatial attributes related to child health and using the up-to-date data.

\section{Conclusion}

This study explores a trend and pattern of four, namely BCG, Diphtheria, Pertussis, Tetanus (DPTs)/Pentavalents dose 3, Polio dose 3, and Measles vaccination coverage from 1993 to 2014 using Bangladesh demographic health survey data. Some significant determinants have also been found for the vaccination coverage distinctly of children age $06-59$ months using the BDHS 2014 data. During the study period, coverage trends showed that the rate of measles vaccine implementation at the 273rd day of a child's age is lower among all analyzed vaccination. The findings of this paper revealed that the administrative region is significantly associated with implementing all four-vaccination coverage. Moreover, being an educated mother's child, better socioeconomic condition, and comparatively older children put some extra advantage of being immunized against DPT, Polio, and Measles diseases. Birth order numbers for BCGs, having media exposure for DPTs, place of residence for Polio, religion for Measles have been found as statistically associated determinants. Finally, these findings recommend that mother's or caregiver's counselling procedures at the time of postnatal care have to continue until children reach their second birthday, and concern officials have to pay more concentration on discussed significant factors, especially in the Sylhet region.

\section{Supplementary Information}

The online version contains supplementary material available at https:/doi. org/10.1186/s12889-021-11576-0.

\section{Additional file 1.}

\section{Acknowledgements}

The authors are grateful to ICF International, Rockville, Maryland, USA, for providing the Bangladesh DHS data sets for this analysis. Authors also grateful to the well-wishers as well as their peers to motivate them for doing this research. We also sincerely thank the two anonymous reviewers, the Editor, and Academic Editor, for their valuable comments and suggestions,

which have been used to improve the manuscript.

\section{Authors' contributions}

MAS, MMH and AR conceptualize the idea; MAS, MMH, SSF, and ZSI design the study; MAS and MMH analyze the data under the guideline of AR; MAS, SSF, ZSI and MMH draft the manuscript; MAS, MMH and AR revised the manuscript based on the review outcomes; AR critically revised the manuscript; the whole study is supervised by AR. The authors read and approved the final manuscript.

\section{Funding}

All authors ensure that this research received no specific grant from any funding agency in the public, commercial or not-for-profit sectors.

\section{Availability of data and materials}

After registration, the data set is available via the following access link http:// dhsprogram.com/data/available-datasets.cfm.

\section{Declarations}

Ethics approval and consent to participate

Not applicable because this study is based on the secondary data. However, the main study follow the proper ethical guidelines.

Consent for publication

Not applicable.

Competing interests

All authors declare that they have no competing interests.

\section{Author details}

'Department of Statistics, Jahangirnagar University, Savar, Dhaka 1342, Bangladesh. ${ }^{2}$ School of Mathematics, Statistics \& Physics, Newcastle University, Newcastle upon Tyne, UK. ${ }^{3}$ Chief Economist's Unit, Bangladesh Bank, Head Office, Dhaka 1000, Bangladesh. ${ }^{4}$ School of Computing, Mathematics and Engineering, Charles Sturt University, Wagga Wagga, NSW 2678, Australia. ${ }^{5}$ Ex-Indoor Medical Officer, Islami Bank Hospital, Mirpur, Dhaka 1000, Bangladesh. ${ }^{6}$ Department of Medicine, University of British Columbia, Vancouver V5Z 1M9, British Columbia, Canada.

Received: 5 November 2020 Accepted: 29 July 2021

Published online: 21 August 2021

\section{References}

1. Keja K, Chan C, Hayden G, Henderson RH. Expanded programme on immunization. World Heal Stat Q. 1988;41:59-63.

2. Adhikary M, Haque R, Tanira S. Determinants of child immunization under expanded programme on immunization (EPI) in a rural setting of Bangladesh. J Dhaka Med Coll. 2015;22(2):201-6. https://doi.org/10.3329/jdmc.v22i2.21543. 
3. Afzal N, Zainab B. Determinants and status of vaccination in Bangladesh. Dhaka Univ J Sci. 2012;60(1):47-51. https://doi.org/10.3329/dujs.v60i1.10336.

4. Alva S, Desia S. Maternal education and child health: is there a strong causal relationship? Demography. 1998;35:71-81.

5. World Health Organisation. State of the world's vaccines and immunization. 2009.

6. de Figueiredo A, Johnston IG, Smith DMD, Agarwal S, Larson HJ, Jones NS. Forecasted trends in vaccination coverage and correlations with socioeconomic factors: a global time-series analysis over 30 years. Lancet Glob Heal. 2016;4(10):e726-35. https://doi.org/10.1016/S2214-109X(16)30167-X.

7. World Health Organization. GVAP 2011-2020, Review and Lessons Learned; Strategic Advisory Group of Experts on Immunization. 2020;1-44.

8. Jamil K, Bhuiya A, Streatfield K, Chakrabarty N. The immunization programme in Bangladesh: impressive gains in coverage, but gaps remain. Health Policy Plan. 1999;14(1):49-58. https://doi.org/10.1093/heapol/14.1.49.

9. Moyazzem Hossain Md, Abdulla F, Karimuzzaman Md, Rahman A. Routine Vaccination Disruption in Low-Income Countries: An Impact of COVID-19 Pandemic. Asia Pac J Public Health. 2020;32(8):509-10. https://doi.org/10.11 77/1010539520957808.

10. World Health Organization (WHO). Global Vaccine Action Plan. Vaccine. 2013.

11. Quaiyum A, Tunon C, Hel Baqui A, Yum ZQ, Khatun J. Impact of national immunization days on polio-related knowledge and practice of urban women in Bangladesh. Health Policy Plan. 1997;12(4):363-71. https://doi. org/10.1093/heapol/12.4.363

12. Expanded Program on Immunization (EPI). Bangladesh EPI Coverage Evaluation Survey 2016. 2017.

13. Liu L, Oza S, Hogan D, Perin J, Rudan I, Lawn JE, et al. Global, regional, and national causes of child mortality in 2000-13, with projections to inform post-2015 priorities: an updated systematic analysis. Lancet. 2015;385(9966): 430-40. https://doi.org/10.1016/S0140-6736(14)61698-6.

14. Ushie BA, Fayehun OA, Ugal DB. Trends and patterns of under-5 vaccination in Nigeria, 1990-2008: what manner of progress? Child Care Health Dev. 2014:40:267-74

15. Calhoun LM, Van Eijk AM, Lindblade KA, Odhiambo FO, Wilson ML, Winterbauer $\mathrm{E}$, et al. Determinants and coverage of vaccination in children in western Kenya from a 2003 cross-sectional survey. Am J Trop Med Hyg. 2014;90(2):234-41. https://doi.org/10.4269/ajtmh.13-0127.

16. Hu Y, Chen Y, Guo J, Tang X, Shen L. Completeness and timeliness of vaccination and determinants for low and late uptake among young children in eastern China. Hum Vaccines Immunother. 2014;10(5):1408-15. https://doi.org/10.4161/hv.28054.

17. Ueda M, Kondo N, Takada M, Hashimoto H. Maternal work conditions, socioeconomic and educational status, and vaccination of children: A community-based household survey in Japan. Prev Med (Baltim). 2014;66: $17-21$.

18. Danis K, Georgakopoulou T, Stavrou T, Laggas D, Panagiotopoulos T. Socioeconomic factors play a more important role in childhood vaccination coverage than parental perceptions: a cross-sectional study in Greece. Vaccine. 2010;28(7):1861-9. https://doi.org/10.1016/j.vaccine.2009.11.078

19. Otieno NA, Nyawanda BO, Audi A, Emukule G, Lebo E, Bigogo G, et al. Demographic, socio-economic and geographic determinants of seasonal influenza vaccine uptake in rural western Kenya, 2011. Vaccine. 2014;32(49): 6699-704. https://doi.org/10.1016/j.vaccine.2013.10.089.

20. Canavan ME, Sipsma HL, Kassie GM, Bradley EH. Correlates of complete childhood vaccination in east African countries. PLoS One. 2014;9(4):e95709. https://doi.org/10.1371/journal.pone.0095709.

21. Wagner KS, Van Wijgerden JCJ, Andrews N, Goulden K, White JM. Childhood vaccination coverage by ethnicity within London between 2006/2007 and 2010/2011. Arch Dis Child. 2014;99(4):348-53. https://doi.org/10.1136/a rchdischild-2013-304388.

22. Pande RP, Yazbeck AS. What's in a country average? Wealth, gender, and regional inequalities in immunization in India. Soc Sci Med. 2003;57(11): 2075-88. https://doi.org/10.1016/S0277-9536(03)00085-6.

23. Reading R, Colver A, Openshaw S, Jarvis S. Do interventions that improve immunisation uptake also reduce social inequalities in uptake? BMJ. 1994; 308(6937):1142-4. https://doi.org/10.1136/bmj.308.6937.1142.

24. Galarce EM, Minsky S, Viswanath K. Socioeconomic status, demographics, beliefs and a(H1N1) vaccine uptake in the United States. Vaccine. 2011; 29(32):5284-9. https://doi.org/10.1016/j.vaccine.2011.05.014.
25. Friederichs V, Cameron JC, Robertson C. Impact of adverse publicity on MMR vaccine uptake: a population based analysis of vaccine uptake records for one million children, born 1987-2004. Arch Dis Child. 2006;91(6):465-8. https://doi.org/10.1136/adc.2005.085944.

26. Mitchell S, Andersson N, Ansari NM, Omer K, Soberanis JL, Cockcroft A. Equity and vaccine uptake: a cross-sectional study of measles vaccination in Lasbela District, Pakistan. BMC Int Health Hum Rights. 2009;9(suppl 1):S7.

27. Le Polain de Waroux O, Schellenberg JRA, Manzi F, Mrisho M, Shirima K, Mshinda $\mathrm{H}$, et al. Timeliness and completeness of vaccination and risk factors for low and late vaccine uptake in young children living in rural southern Tanzania. Int Health. 2013;5(2):139-47. https://doi.org/10.1093/inthealth/iht006.

28. Herliana P, Douiri A. Determinants of immunisation coverage of children aged 12-59 months in Indonesia: a cross-sectional study. BMJ Open. 2017;7:1-14.

29. Oleribe O, Kumar V, Awosika-Olumo A, Taylor-Robinson SD. Individual and socioeconomic factors associated with childhood immunization coverage in Nigeria. Pan Afr Med J. 2017;26:1-14.

30. Meleko A, Geremew M, Birhanu F. Assessment of child immunization coverage and associated factors with full vaccination among children aged 12-23 months at Mizan Aman Town, Bench Maji Zone, Southwest Ethiopia. Int J Pediatr. 2017;2017:7976587.

31. Rutstein SO, Rojas G. Guide to DHS Statistics: Demographic and Health Surveys Methodology. 2006.

32. Uddin MJ, Larson CP, Oliveras E, Khan Al, Quaiyum MA, Saha NC. Child immunization coverage in urban slums of Bangladesh: impact of an intervention package. Health Policy Plan. 2010;25(1):50-60. https://doi.org/1 0.1093/heapol/czp041

33. Sheikh N, Sultana M, Ali N, Akram R, Mahumud R, Asaduzzaman M, et al. Coverage, timelines, and determinants of incomplete immunization in Bangladesh. Trop Med Infect Dis. 2018;3:1-14.

34. Rahman A, Reza AAS, Bhuiyan BA, Alam N, Dasgupta SK, Mostari S, et al. Equity and determinants of routine child immunisation programme among tribal and non-tribal populations in rural Tangail subdistrict, Bangladesh: a cohort study. BMJ Open. 2018;8:e022634.

35. National Institute of Population Research and Trainning (NIPORT). Bangladesh Demographic and Health Survey 1993-1994. 1994.

36. National Institute of Population Research and Trainning (NIPORT). Bangladesh Demographic and Health Survey 1996-1997. 1997.

37. National Institute of Population Research and Trainning (NIPORT). Bangladesh Demographic and Health Survey 1999-2000. 2001.

38. National Institute of Population Research and Trainning (NIPORT). Bangladesh Demographic and Health Survey 2004. 2004.

39. National Institute of Population Research and Trainning (NIPORT). Bangladesh Demographic and Health Survey 2007. 2009.

40. National Institute of Population Research and Trainning (NIPORT). Bangladesh Demographic and Health 2011. 2013.

41. National Institute of Population Research and Trainning (NIPORT). Bangladesh Demograph and Health Survey 2014. 2016.

42. Rahman A. Estimating small area health-related characteristics of populations: a methodological review. Geospat Health. 2017;12(1):3-14. https://doi.org/10.4081/gh.2017.495

43. Lo Vecchio A, Cambriglia MD, Fedele MC, Basile FW, Chiatto F. Miraglia del Giudice $M$, et al. determinants of low measles vaccination coverage in children living in an endemic area. Eur J Pediatr. 2019;178(2):243-51. https:// doi.org/10.1007/s00431-018-3289-5.

44. Sarker AR, Akram R, Ali N, Sultana M. Coverage and factors associated with full immunisation among children aged 12-59 months in Bangladesh: insights from the nationwide cross-sectional demographic and health survey. BMJ Open. 2019;9(7):e028020. https://doi.org/10.1136/bmjopen-2018-028020.

45. Jahan Y, Moriyama M, Rahman MM, Shahid AS, Bin MS, Rahman A, et al Changing trends in measles vaccination status between 2004 and 2014 among children aged 12-23 months in Bangladesh. Trop Med Int Heal. 2020;25:475-82

46. Acharya $K$, Paudel YR, Dharel D. The trend of full vaccination coverage in infants and inequalities by wealth quintile and maternal education: analysis from four recent demographic and health surveys in Nepal. BMC Public Health. 2019;19(1):1673. https://doi.org/10.1186/s12889-019-7995-3.

\section{Publisher's Note}

Springer Nature remains neutral with regard to jurisdictional claims in published maps and institutional affiliations. 